\title{
A correlation between the number of satellites and the baryonic bulge-to-total ratio extending beyond the Local Group
}

\author{
Behnam Javanmardi ${ }^{1 \star}$ and Pavel Kroupa ${ }^{2,3}$ \\ ${ }^{1}$ LESIA, Paris Observatory, PSL Univ., CNRS, Sorbonne Univ., Univ. Paris Diderot, 5 Place Jules Janssen, 92195 Meudon, France \\ ${ }^{2}$ Helmholtz Institut für Strahlen- und Kernphysik (HISKP), University of Bonn, Nussalle 14-16, 53121 Bonn, Germany \\ ${ }^{3}$ Charles University, Faculty of Mathematics and Physics, Astronomical Institute, V Holeŝovivckách 2, 180 00 Praha 8, Czech Republic
}

\section{INTRODUCTION}

Until recently, the halos of only the Milky Way (MW) and Andromeda (M31) galaxies have been observed deep enough to enable studying different properties of their dwarf satellites (e.g. Grebel 1997; McConnachie 2012; Collins et al. 2015) and to test the expectations of galaxy formation and evolution scenarios down to small scales. The observations of these two galaxies, and the Local Group (LG) in general, has led to some of the long lasting challenges for the standard Lambda-Cold-Dark-Matter $(\Lambda \mathrm{CDM})$ cosmological model, viz: the missing satellites (Klypin et al. 1999), the too-big-to-fail (Boylan-Kolchin et al. 2011), and the disk of satellites (Kroupa et al. 2005; Pawlowski \& Kroupa 2013) problems. The numerous observational, theoretical, and computational studies aiming at solving these issues have not reached a consensus so far (see e.g. Bullock \& Boylan-Kolchin 2017, for a review). These problems required systematic and deep surveys for finding more dwarf satellites outside the LG. In the recent years, and thanks to various dedicated deep observations (e.g. Geha et al. 2017; Greco et al. 2018), other galaxy groups are also turning into very useful laboratories for investigating the small scale pre-

^ E-mail: behnam.javanmardi@obspm.fr dictions ${ }^{1}$ of the $\Lambda$ CDM model, and for learning about galaxy formation and evolution in general.

Another interesting feature of the LG that motivated dedicated studies (such as Javanmardi et al. 2016; LópezCorredoira \& Kroupa 2016; Henkel et al. 2017) is an observed correlation between the number of dwarf satellites, $N_{\text {sat }}$, and bulge mass $\left(M_{\text {bulge }}\right)$ of M33, MW, and M31 (Kroupa et al. 2010). This correlation appeared to be a puzzling one due to the following two reasons: i) in the $\Lambda \mathrm{CDM}$ model, $N_{\text {sat }}$ is expected to correlate with only the mass of the dark matter halo, hence with the rotation velocity, of the disk galaxy, and ii) galaxies with similar rotation velocities (e.g. M31 and M101, Faber \& Gallagher 1979) but with very different $M_{\text {bulge }}$ are observed in the Universe. Therefore, unlike the expected correlation between $N_{\text {sat }}$ and rotation velocity, a correlation between $N_{\text {sat }}$ and $M_{\text {bulge }}$ was not expected.

To quantify the expectations from the $\Lambda$ CDM model for this correlation, Javanmardi et al. (2019) used data from the Millennium-II simulation (Boylan-Kolchin et al. 2009) with the semi-analytic galaxy formation model of Guo et al.

\footnotetext{
1 We note though that the disk-of-satellites problem is not a small scale problem as it refers to the distribution of baryonic matter on scales of hundreds of kpc about major host galaxies.
} 
(2011) and reported three main results: i) for a sample of disk galaxies with a wide mass range, a weak correlation (with a large scatter) between $N_{\text {sat }}$ and $M_{\text {bulge }}$ emerges which reflects the seemingly independent correlations between these two quantities and the mass of the disk galaxy, ii) for disk galaxies with similar masses or rotation velocities, no correlation between $N_{\text {sat }}$ and $M_{\text {bulge }}$ is found, and iii) when normalizing $M_{\text {bulge }}$ with the mass of the disk galaxy, i.e. when considering $B / T$ ratios, no correlation is found between $N_{\text {sat }}$ and $B / T$ of disk galaxies with stellar mass $M_{\star} \approx 10^{10}-10^{11} M_{\odot}$ in the $\Lambda \mathrm{CDM}$ model.

Recently, observations of the galaxies M81, Centaurus A (Cen A), M94, and M101 (at distances of about 3.7, 3.8, 4.2 , and $7 \mathrm{Mpc}$, respectively) have become deep enough to enable studying their satellite properties and their relation to their hosts (Chiboucas et al. 2013; Crnojević et al. 2019; Müller et al. 2019; Smercina et al. 2018; Carlsten et al. 2019b; Bennet et al. 2019). In this contribution, we use the published data from these galaxy groups, together with the available data from M33, MW, and M31, to investigate the $N_{\text {sat }}$ vs. $B / T$ correlation.

\section{DATA AND ANALYSIS}

In this section, we first introduce the data we use in our analysis from different studies published in the literature. See Table 1 for a summary. We then apply a simple correlation analysis and present the results.

\subsection{Number of dwarf satellites}

The information about the satellite populations of the galaxies in our study are as follows:

- The LG galaxies: we refer to McConnachie (2012) and McConnachie et al. (2018), for the information about MW and M31 satellites, respectively ${ }^{2}$.

- M81: using the Canada-France-Hawaii Telescope (CFHT) and Hubble Space Telescope (HST) observations, Chiboucas et al. (2009) and Chiboucas et al. (2013) have discovered and confirmed 14 new dwarf galaxies in the M81 group. Their survey covers at least $\approx 300 \mathrm{kpc}$ projected distance around M81 (and in some directions more than twice that, see figure 27 of Chiboucas et al. 2013). They increased the number of known members of this group to 36 with its faintest dwarf having an r-band absolute magnitude of $M_{\mathrm{r}}=-$ $6.8\left(M_{\mathrm{V}} \approx-8\right.$, Chiboucas et al. 2013). See their Table 4 for a full list of information. It is worth noting that we do not include two tidal dwarf galaxy candidates of M81 (namely A0952 and Garlnd, Karachentsev et al. 2002; Makarova et al. 2002) in our analysis due to uncertainties on their nature. We also note that we treat M82 as a satellite galaxy of M81 (rather than considering it separately) because M82 (being less than $100 \mathrm{kpc}$ in projection away from M81) shows clear signs of disturbance and is undergoing a star-burst, indicating strong interactions between the two galaxies ${ }^{3}$.

2 Note that a possible, but not confirmed, satellite of M33 is reported by Martin et al. (2009) with $M_{\mathrm{V}}=-6.5$.

3 We note that M33 shows no signs of interaction, i.e. is close to
- Cen A: Crnojević et al. (2019) and Müller et al. (2019) have used the Panoramic Imaging Survey of Centaurus and Sculptor (PISCeS), the HST, and the ESO Very Large Telescope (VLT) to confirm the dwarf satellite candidates of Cen A, most of which were identified by Crnojević et al. (2014), Crnojević et al. (2016), Müller et al. (2015), and Müller et al. (2017a). Together, Crnojević et al. (2019) and Müller et al. (2019) report in total 31 confirmed dwarf satellites for Cen A with the faintest having a $\mathrm{V}$-band absolute magnitude of $M_{\mathrm{V}}=-7.8 \mathrm{mag}$. According to Müller et al. (2019), the satellite population of Cen A is complete within its $200 \mathrm{kpc}$ projected distance, while two of its already confirmed satellites, namely KK 211 and ESO 325-011, are at projected distances of 240 and $246 \mathrm{kpc}$, respectively (Crnojević et al. 2019).

- M94: using deep Hyper Suprime-Cam (HSC) observations, Smercina et al. (2018) performed a survey of the field of M94 down to $M_{\mathrm{V}}=-9.1 \mathrm{mag}$ finding only two satellites for this galaxy. Regrettably, their survey covers only out to $150 \mathrm{kpc}$ projected distance and while we discuss this galaxy in our study, we do not include it in our correlation analysis.

- M101: Danieli et al. (2017) and Bennet et al. (2019) used deep HST observations and measured the Tip of the Red Giant Branch (TRGB) distances, and Carlsten et al. (2019b) have used the CFHT data and measured surface brightness fluctuations distances to satellite candidates around M101. These studies confirmed in total 8 satellites for this galaxy with the faintest having a V-band absolute magnitude of $M_{\mathrm{V}}=-8.2 \mathrm{mag}$. Most of these candidates have been found in a wide survey using also the CFHT by Bennet et al. (2017) and some of them by Merritt et al. (2014), Javanmardi et al. (2016), and Müller et al. (2017b). These surveys cover almost isotropically the $260 \mathrm{kpc}$ virial radius of M101 (see figure 1 in Carlsten et al. 2019b).

The latter galaxy group imposes a magnitude limit on our analysis and to be able to compare the satellite populations of all galaxies, we only count the satellites with $M_{\mathrm{V}} \leq-8.2 \mathrm{mag}$. In addition, we will perform our correlation analysis (Section 2.3) with two projected distance limits; first considering only the satellites within $200 \mathrm{kpc}$ projected distance to their hosts, and second increasing the projected distance limit to $250 \mathrm{kpc}$ by assuming that the satellite population of Cen A is complete-enough out to that distance, see the last columns of Table 1 where $N_{\text {sat }}^{200}$ and $N_{\text {sat }}^{250}$ represent the number of satellites within 200 and $250 \mathrm{kpc}$ of each galaxy, respectively.

\section{$2.2 \quad$ Bulge-to-total mass ratios}

The information about the $B / T$ ratios of the galaxies in our study are as follows:

- For MW, M31, M81, Cen A, and M101, stellar mass and $B / T$ values are directly adopted from Bell et al. (2017). See their table 1 for a list of references and other measurements.

- For M33, using $M_{\text {bulge }}=1.14( \pm 0.14) \times 10^{8} M_{\odot}$ reported by Seigar $(2011)$, and adopting a total mass of $\approx 10^{10} M_{\odot}$ reported by Corbelli (2003), we obtain $B / T=0.01 \pm 0.001$.

being nearly isolated, and therefore we consider it as a separate galaxy and not as a satellite of Andromeda. 


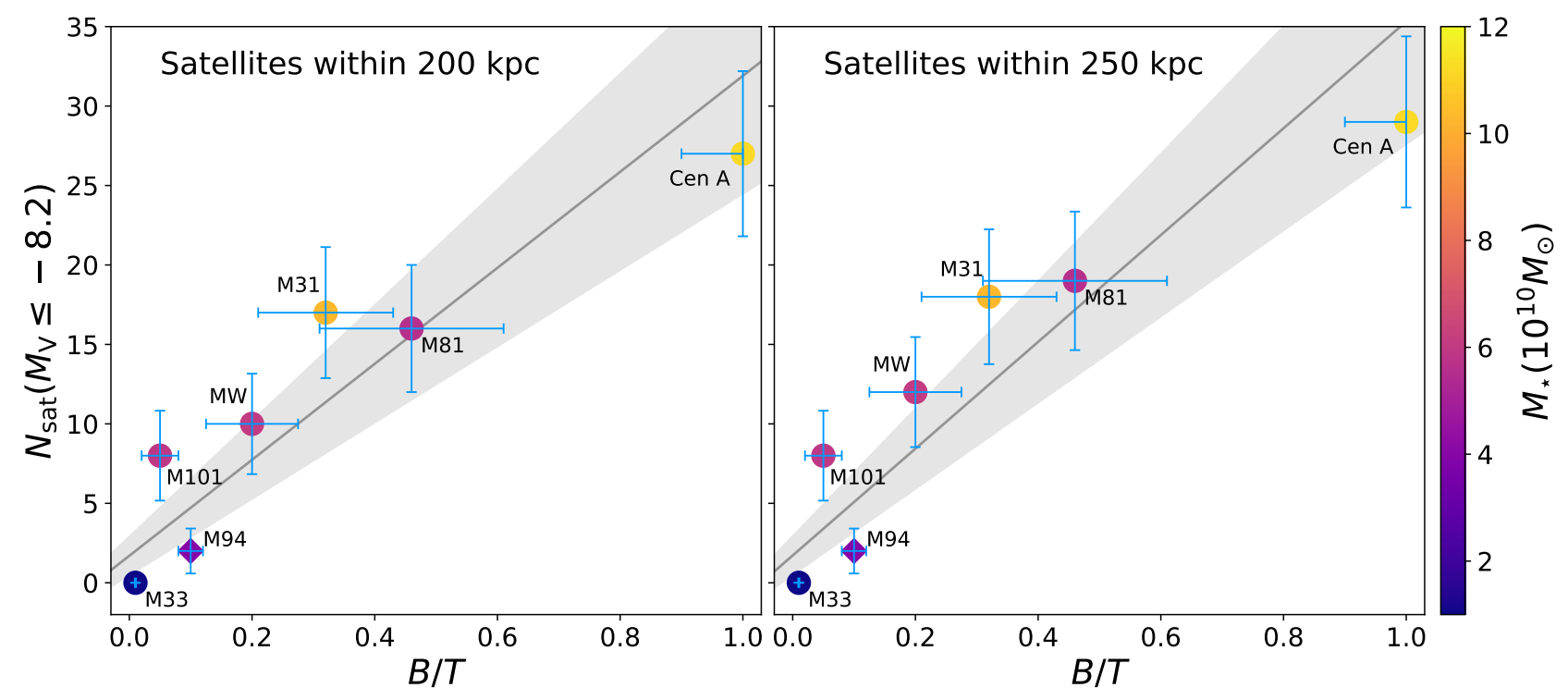

Figure 1. Number of satellites, $N_{\text {sat }}$ (with $M_{\mathrm{V}} \leq-8.2$ ) vs. bulge-to-total baryonic mass ratio, $B / T$. The left and right panels correspond to considering satellites within 200 and $250 \mathrm{kpc}$ of their host galaxies, respectively. The color code is $M_{\star}$, the solid grey lines are the results of linear fits taking into account uncertainties on both axes, and the shaded regions are the $1 \sigma$ uncertainty of the fitted results. For both of the analyses, the probability that the correlation arises by chance is only 0.5 percent. Since the current surveys cover only $150 \mathrm{kpc}$ projected distance of M94 (shown by a diamond in this figure), this galaxy is excluded from both the fitting and the correlation analyses. Adding M94 would increase the significance of the correlation.

Table 1. The data used in this study and their references. See Section 2.

\begin{tabular}{lcccc} 
Galaxy & $M_{\star}\left(10^{10} M_{\odot}\right)$ & $B / T$ & $N_{\text {sat }}^{200}$ & $N_{\text {sat }}^{250}$ \\
\hline MW & $6.1(\mathrm{a})$ & $0.2 \pm 0.075(\mathrm{a})$ & $10(\mathrm{~b})$ & $12(\mathrm{~b})$ \\
M31 & $10.3(\mathrm{a})$ & $0.32 \pm 0.11(\mathrm{a})$ & $17(\mathrm{c})$ & $18(\mathrm{c})$ \\
M33 & $1(\mathrm{~d})$ & $0.01 \pm 0.001(\mathrm{~d}, \mathrm{e})$ & 0 & 0 \\
M81 & $5.6(\mathrm{a})$ & $0.46 \pm 0.15(\mathrm{a})$ & $16(\mathrm{f})$ & $19(\mathrm{f})$ \\
Cen A & $11.2(\mathrm{a})$ & $1.0_{-0.1}^{+0.0}(\mathrm{a})$ & $27(\mathrm{~g}, \mathrm{~h})$ & $29(\mathrm{~g}, \mathrm{~h})$ \\
M94 & $4(\mathrm{i})$ & $0.1 \pm 0.02(\mathrm{j}, \mathrm{k})$ & $2^{\dagger}(\mathrm{i})$ & $2^{\dagger}(\mathrm{i})$ \\
M101 & $5.9(\mathrm{a})$ & $0.05 \pm 0.03(\mathrm{a})$ & $8(\mathrm{l}, \mathrm{m})$ & $8(1, \mathrm{~m})$ \\
\hline
\end{tabular}

(a) Bell et al. (2017); (b) McConnachie (2012); (c) McConnachie et al. (2018); (d) Corbelli (2003); (e) Seigar (2011); (f) Chiboucas et al. (2013); (g) Crnojević et al. (2019); (h) Müller et al. (2019); (i) Smercina et al. (2018); (j) Moellenhoff et al. (1995); (k) Jałocha et al. (2010); (l) Carlsten et al. (2019b); (m) Bennet et al. (2019).

( $\dagger$ ) Note that the field of M94 is surveyed only out to $150 \mathrm{kpc}$ projected distance, and while we show it in Figure 1, we do not include it in our correlation analysis.

- For M94, we adopt the mean (and its standard error) of the $M_{\text {bulge }}$ values reported in Moellenhoff et al. (1995) and Jałocha et al. $(2010) ; M_{\text {bulge }}=4.54( \pm 0.86) \times 10^{9} M_{\odot}$. Considering a stellar mass of $4 \times 10^{10} M_{\odot}$ (Smercina et al. 2018), we obtain a $B / T=0.1 \pm 0.002$ for M94.

See the second and third columns of Table 1 for a summary.

\subsection{The correlation}

The data listed in Table 1 are visualized in Figure 1 by plotting $N_{\mathrm{sat}}$ vs. $B / T$. Each galaxy is labeled on this figure and the color code represents the $M_{\star}$. The uncertainties on $B / T$ are values from Table 1 , and we consider an uncertainty of $\pm \sqrt{N_{\text {sat }}}$ for the number of satellites. The left and right panels correspond to considering satellites within 200 and $250 \mathrm{kpc}$ of their host galaxies, respectively. The solid grey lines are the result of linear least squared fits to the data: $N_{\text {sat }}^{200}=30.2( \pm 6.2) B / T+1.7( \pm 1.3)$, and $N_{\text {sat }}^{250}=33.6( \pm 6.5) B / T+$ $1.7( \pm 1.3)$. In the fitting procedure, we take both $N_{\text {sat }}$ and $B / T$ uncertainties into account. The shaded regions reflect the $1 \sigma$ uncertainty of the fitting results. However, we note that we do not aim to present a formula for $N_{\text {sat }}$ vs. $B / T$ and the purpose of the fitting is to better see the trend presented by these data.

Our main finding is that using these data we measure the linear correlation coefficient for $N_{\text {sat }}$ vs. $B / T$ to be $r=0.94$ and the probability that this correlation arises by chance to have a $p$-value $=0.005$, for both of the projected distance conditions ${ }^{4}$. In other words, the data yield a 99.5 percent (around $3 \sigma$ ) significant correlation between $N_{\text {sat }}$ and $B / T$. We note that M94 is excluded from both the linear fitting and the correlation measurement.

4 The $r$ and the $p$ values for the two projected distance conditions differ only after the third decimal places, not reported here. 


\section{DISCUSSION AND CONCLUDING REMARKS}

Figure 1 can be compared with the right panel of figure 4 in Javanmardi et al. (2019) which shows $N_{\text {sat }}$ vs. $B / T$ for a sample of more than 6000 disk galaxies with stellar masses between 1.2 and $26.3 \times 10^{10} M_{\odot}$ from the Millennium-II simulation (Boylan-Kolchin et al. 2009). This mass range almost encompasses that of the spiral galaxies in our study. Javanmardi et al. (2019) measure a linear correlation coefficient of only $r=0.13$ for $N_{\text {sat }}$ vs. $B / T$, being consistent with no correlation between these two quantities in the $\Lambda$ CDM model.

This can be understood also qualitatively by noting that in the $\Lambda$ CDM model, $N_{\text {sat }}$ is directly related to dark matter halo mass; the heavier the halo, the larger the number of accreted subhalos (top left panel of figure 3 in Javanmardi et al. 2019), and heavier halos accrete also more baryonic mass forming galaxies with statistically larger stellar masses. In addition, in this model, galaxies with larger stellar masses are found to be more likely to grow heavier bulges (figure 2 in Javanmardi et al. 2019). Therefore, in the $\Lambda$ CDM model, $N_{\text {sat }}$ is weakly correlated with $M_{\text {bulge }}$, but not with $B / T$. This certainly requires further detailed studies of bulge formation and its possible connection with large-scale environment in this model (Romano-Díaz et al. 2017, Tavasoli et al. in prep.).

An interesting point in the current data is that while M81, M101, and the MW have very similar stellar masses, they have different $N_{\text {sat }}$ and very different $B / T$ ratios, implying a lack of correlation between these two quantities and stellar mass.

We note that Cen A has a few features which makes it distinct with respect to the other galaxies in our analysis. It is not a disk-galaxy (with $B / T=1.0$ ), it has an active nucleus, and it has a perturbed structure. Assuming that these have a significant impact on its satellite population, remeasuring the $N_{\text {sat }}$ vs. $B / T$ correlation without Cen A gives $r=0.93$ and $p$-value $=0.02$, not changing our main result.

In addition, it should be emphasised that as mentioned earlier, M94 is not included in our correlation analysis because its field is surveyed out to only $150 \mathrm{kpc}$ projected distance. Assuming that future surveys do not change the number of satellites of M94 significantly, adding it to the analysis would increase the significant of the correlation to well above the $3 \sigma$ confidence level. Actually, even if future surveys increase the number of confirmed satellites of M94 by a factor of 3 or 4 , the significance of the $N_{\text {sat }}$ vs. $B / T$ correlation would increase even further. It is therefore very important to conduct deeper and wider surveys in the field of this particular galaxy.

In a very recent study, Carlsten et al. (2019a) have reported the detection of satellite galaxy candidates around 10 galaxies including M104 (a.k.a. the Sombrero galaxy, at a distance of around $9.5 \mathrm{Mpc}$ ). They report in total 27 satellite candidates within $150 \mathrm{kpc}$ projected distance of this galaxy $^{5}$. With $B / T>0.7$ (Gadotti \& Sánchez-Janssen 2012), this galaxy can add another point to the large $B / T$ values

5 Also confirming the low surface brightness galaxies found by the Dwarf Galaxy Survey with Amateur Telescopes (DGSAT, Javanmardi et al. 2016) within $80 \mathrm{kpc}$ projected distance of M104. of the $N_{\text {sat }}$ vs. $B / T$ relation. Carlsten et al. (2019a) also report in total 21 satellite candidates for NGC 4565 that has a $B / T=0.25$ (Bell et al. 2017). However, follow up distance measurements for their satellite candidates are essential before including these two galaxies in the analysis.

As a final remark, it is also worth noting other reported interesting properties of the non-LG galaxies in our study. Chiboucas et al. (2013) found that M81 has a disk of satellites similar to those of MW (Kroupa et al. 2005; Pawlowski \& Kroupa 2013) and M31 (Metz et al. 2007; Hammer et al. 2013; Ibata et al. 2014). In addition, Müller et al. (2018) reported a similar structure around the Cen A galaxy. Smercina et al. (2018) and Bennet et al. (2019) conclude that M94 and M101 have sparse satellite populations, not fulfilling the expectations from the $\Lambda \mathrm{CDM}$ model. Bennet et al. (2019) suggests a link between the properties of these galaxy groups and their environments reporting that galaxies in more tidally active environments tend to have a larger $N_{\text {sat }}$ (see their figure 8).

The latter point is in line with an alternative scenario by Kroupa (2015) arguing that if most of the observed satellites are ancient tidal dwarf galaxies, then a correlation between $N_{\text {sat }}$ and $B / T$ can emerge because bulge growth is enhanced significantly in galaxy-galaxy encounters. This would also explain the observed preferred distribution of satellites in rotating disks (Pawlowski et al. 2013; Kroupa 2012). This scenario also requires further detailed and quantitative studies (see e.g. Combes 2016; Bílek et al. 2018; Banik et al. 2018).

Whatever the correct underlying theory will turn out to be, and while further observational studies of these and other galaxy groups are necessary, the main finding of our study is that the current data show a strong and significant correlation between $N_{\text {sat }}$ and $B / T$ in the most well-studied galaxy groups in the Local Volume.

\section{ACKNOWLEDGEMENTS}

We thank the anonymous referee for the constructive comments. The research leading to these results has received funding from the European Research Council (ERC) under the European Union's Horizon 2020 research and innovation program (grant agreement No. 695099).

\section{REFERENCES}

Banik I., O'Ryan D., Zhao H., 2018, MNRAS, 477, 4768

Bell E. F., Monachesi A., Harmsen B., de Jong R. S., Bailin J., Radburn-Smith D. J., D'Souza R., Holwerda B. W., 2017, ApJ, 837, L8

Bennet P., Sand D. J., Crnojević D., Spekkens K., Zaritsky D., Karunakaran A., 2017, ApJ, 850, 109

Bennet P., Sand D. J., Crnojević D., Spekkens K., Karunakaran A., Zaritsky D., Mutlu-Pakdil B., 2019, arXiv e-prints, p. arXiv:1906.03230

Bílek M., Thies I., Kroupa P., Famaey B., 2018, A\&A, 614, A59

Boylan-Kolchin M., Springel V., White S. D. M., Jenkins A., Lemson G., 2009, MNRAS, 398, 1150

Boylan-Kolchin M., Bullock J. S., Kaplinghat M., 2011, MNRAS, 415, L40

Bullock J. S., Boylan-Kolchin M., 2017, ARA\&A, 55, 343

Carlsten S. G., Greco J. P., Beaton R. L., Greene J. E., 2019a, arXiv e-prints, p. arXiv:1909.07389 
Carlsten S. G., Beaton R. L., Greco J. P., Greene J. E., 2019b, ApJ, 878, L16

Chiboucas K., Karachentsev I. D., Tully R. B., 2009, AJ, 137, 3009

Chiboucas K., Jacobs B. A., Tully R. B., Karachentsev I. D., 2013, The Astronomical Journal, 146, 126

Collins M. L. M., et al., 2015, ApJ, 799, L13

Combes F., 2016, in Laurikainen E., Peletier R., Gadotti D., eds, Astrophysics and Space Science Library Vol. 418, Galactic Bulges. p. 413 (arXiv:1501.03603), doi:10.1007/978-3-31919378-6_15

Corbelli E., 2003, MNRAS, 342, 199

Crnojević D., et al., 2014, ApJ, 795, L35

Crnojević D., et al., 2016, ApJ, 823, 19

Crnojević D., et al., 2019, ApJ, 872, 80

Danieli S., van Dokkum P., Merritt A., Abraham R., Zhang J., Karachentsev I. D., Makarova L. N., 2017, The Astrophysical Journal, 837, 136

Faber S. M., Gallagher J. S., 1979, ARA\&A, 17, 135

Gadotti D. A., Sánchez-Janssen R., 2012, MNRAS, 423, 877

Geha M., et al., 2017, ApJ, 847, 4

Grebel E. K., 1997, in Schielicke R. E., ed., Reviews in Modern Astronomy Vol. 10, Reviews in Modern Astronomy. pp 29-60

Greco J. P., et al., 2018, ApJ, 857, 104

Guo Q., et al., 2011, MNRAS, 413, 101

Hammer F., Yang Y., Fouquet S., Pawlowski M. S., Kroupa P., Puech M., Flores H., Wang J., 2013, MNRAS, 431, 3543

Henkel C., Javanmardi B., Martínez-Delgado D., Kroupa P., Teuwen K., 2017, A\&A, 603, A18

Ibata R. A., Ibata N. G., Lewis G. F., Martin N. F., Conn A., Elahi P., Arias V., Fernando N., 2014, ApJ, 784, L6

Jałocha J., Bratek L., Kutschera M., 2010, Acta Physica Polonica Series B, 41, 1383

Javanmardi B., et al., 2016, A\&A, 588, A89

Javanmardi B., Raouf M., Khosroshahi H. G., Tavasoli S., Mueller O., Molaeinezhad A., 2019, The Astrophysical Journal, 870, 50

Karachentsev I. D., et al., 2002, A\&A, 383, 125

Klypin A., Kravtsov A. V., Valenzuela O., Prada F., 1999, ApJ, 522,82

Kroupa P., 2012, Publ. Astron. Soc. Australia, 29, 395

Kroupa P., 2015, Canadian Journal of Physics, 93, 169

Kroupa P., Theis C., Boily C. M., 2005, A\&A, 431, 517

Kroupa P., et al., 2010, A\&A, 523, A32

López-Corredoira M., Kroupa P., 2016, ApJ, 817, 75

Makarova L. N., et al., 2002, A\&A, 396, 473

Martin N. F., et al., 2009, ApJ, 705, 758

McConnachie A. W., 2012, AJ, 144, 4

McConnachie A. W., et al., 2018, The Astrophysical Journal, 868, 55

Merritt A., van Dokkum P., Abraham R., 2014, ApJ, 787, L37

Metz M., Kroupa P., Jerjen H., 2007, MNRAS, 374, 1125

Moellenhoff C., Matthias M., Gerhard O. E., 1995, A\&A, 301, 359

Müller O., Jerjen H., Binggeli B., 2015, A\&A, 583, A79

Müller O., Jerjen H., Binggeli B., 2017a, A\&A, 597, A7

Müller O., Scalera R., Binggeli B., Jerjen H., 2017b, A\&A, 602, A119

Müller O., Pawlowski M. S., Jerjen H., Lelli F., 2018, Science, 359,534

Müller O., Rejkuba M., Pawlowski M. S., Ibata R., Lelli F., Hilker M., Jerjen H., 2019, A\&A, 629, A18

Pawlowski M. S., Kroupa P., 2013, MNRAS, 435, 2116

Pawlowski M. S., Kroupa P., Jerjen H., 2013, MNRAS, 435, 1928

Romano-Díaz E., Garaldi E., Borzyszkowski M., Porciani C., 2017, MNRAS, 469, 1809

Seigar M. S., 2011, ISRN Astronomy and Astrophysics, 2011, 725697
Smercina A., Bell E. F., Price P. A., D'Souza R., Slater C. T., Bailin J., Monachesi A., Nidever D., 2018, ApJ, 863, 152 Tavasoli S., Javanmardi B., et al. in prep.

This paper has been typeset from a $\mathrm{T}_{\mathrm{E}} \mathrm{X} / \mathrm{LAT} \mathrm{E} \mathrm{X}$ file prepared by the author. 